British Journal of Education, Society \&
Behavioural Science
$17(1): \begin{gathered}1-15,2016, \text { Article no.BJESBS.26393 } \\ \text { ISSN: 2278-0998 }\end{gathered}$
SCIENCEDOMAIN international
www.Sciencedomain.org

\title{
Demographic and Lifestyle Correlates of School Attendance, English and Maths Attainment, and the Occurrence of Behavioural Sanctions in British Secondary School Children
}

\author{
Gareth Richards ${ }^{1 *}$ and Andrew P. Smith ${ }^{1}$ \\ ${ }^{1}$ Centre for Occupational and Health Psychology, School of Psychology, Cardiff University, \\ 63 Park Place, Cardiff, CF10 3AS, UK.
}

\begin{abstract}
Authors' contributions
Author APS designed the study and supervised the project. Author GR carried out the statistical analysis and interpretation, and drafted the original manuscript. Author APS revised the initial draft for important intellectual content, and both authors read and approved the final manuscript before submission.

Article Information

DOI: 10.9734/BJESBS/2016/26393

Editor(s):

(1) Chih-Wei Pai, Taipei Medical University, Taiwan ROC.

Reviewers:

(1) Adenike Emeke, University of Ibadan, Nigeria. (2) Gina Maria Quinás Tomé, Instituto de Saúde Ambiental, University of Lisbon, Portugal.

(3) Maria G. Fabregas Janeiro, University of California, USA.

(4) Yunus Dogan, Fırat University, Turkey. Complete Peer review History: http://sciencedomain.org/review-history/15325

Original Research Article

Received $14^{\text {th }}$ April 2016

Accepted $25^{\text {th }}$ May 2016

Published $9^{\text {th }}$ July 2016
\end{abstract}

\section{ABSTRACT}

Aims: Performance in school is known to predict a number of important outcomes in later life (e.g. socioeconomic status). For this reason it is considered useful to identify aspects of demography and lifestyle that are associated with low school attendance, low academic attainment, and high occurrences of problem behaviour.

Study Design: The current study utilised a cross-sectional design; analyses were performed twice due to the availability of two cross-sections of data from the same sample.

Place and Duration of Study: Data from the current study were collected from three secondary

*Corresponding author: E-mail: RichardsG6@cardiff.ac.uk; 
schools in Cornwall, UK. The first cross-section was collected in December, 2012, and the second was collected in June, 2013.

Methodology: The School Information Management System was used to obtain data relating to demography (sex, school and year group attended, eligibility/ineligibility to receive free school meals, and presence/absence of a special educational needs status) and school performance (attendance, attainment at Key Stage 3/Key Stage 4 English and maths, and occurrence of behavioural sanctions). Lifestyle factors (number of sleep hours, and frequency of exercise participation) were assessed via pen and paper questionnaire. Chi-square, chi-square tests of linear association, and between-subjects t-tests were used to establish whether the school performance outcomes were associated with the demographic and lifestyle variables. These analyses were then followed-up with binary logistic regression, to determine whether the observed effects were independent of one another.

Results: Low school performance was consistently associated with male sex, school and year group attended, special educational needs status, eligibility to receive free school meals, low sleep hours, and infrequent exercise participation. In addition, below average school attendance was itself predictive of low English and maths attainment, and of a high occurrence of behavioural sanctions. The majority of effects observed were significant at both the univariate and multivariate levels.

Conclusions: The identification of demographic and lifestyle correlates of school performance may be useful for detecting at-risk individuals who might benefit from interventions. If such interventions were to be effective, the associated reductions in future unemployment and criminality could be beneficial to society as a whole.

Keywords: Adolescent behavior; attainment; demography; education; exercise; sleep.

\section{INTRODUCTION}

The degree to which a child achieves at school can have a considerable impact on a range of later-life outcomes [1]. It is therefore of interest to establish demographic and lifestyle factors that may be related to school performance. The current study aimed to investigate four such outcomes: school attendance, English attainment, maths attainment, and the occurrence of behavioural sanctions.

Low school attendance has been a particular concern in the UK for a number of years. According to Taylor [2], there were 57 million days of school missed in 2009/2010, and of children who miss $50 \%$ of school, only $3 \%$ achieve the government target of five or more GCSEs with grades $A^{*}-C$ including English and maths. In addition, children with low attendance are less likely to be in employment, further education or training once leaving school [2]. Though unemployment can be a major problem in itself, it is also associated with a number of undesirable outcomes, such as poor health and suicide [3], and criminal behaviour [4]. Furthermore, it can have considerable knock-on effects at the societal level [5].

In addition to the above, low school attendance is a strong predictor of low educational attainment
[6]. This is of particular concern considering that attainment is known to predict future outcomes, such as career prospects and earning potential [7]. Low attendance and attainment are also known to co-occur with a range of parental variables, including low socioeconomic status (SES), conflict, neglect, criminal record, and mental illness $[8,9,10]$. Moreover, research has shown that family circumstances, and parental interest and attitudes towards education are stronger predictors of a child's school attainment than are school factors, such as extra resources being made available in areas of high need [11].

A further concern is that low academic attainment is associated with antisocial behaviour and delinquency [12]. Disruptive behaviour in school is a problem for several reasons. Firstly, it can be distracting, making it difficult for teachers to teach, as well as for other students to learn. This can cause collateral harm to students' academic achievement, and damage the reputation of the school. Problem behaviour is also associated with future criminality [13], making it a variable of particular societal interest.

Due to the above concerns, the current paper presents data from the Cornish Academies Project relating to school attendance, Key Stage 
3/Key Stage 4 (KS3/KS4) English and maths attainment, and the occurrence of problem behaviour. The aim is to examine how certain aspects of demography and lifestyle could be associated with below average school performance. Firstly, it was hypothesised that low school performance outcomes would be associated with the following demographic factors: male sex, school year, eligibility to receive free school meals (FSM), presence of a special educational needs (SEN) status. Secondly, it was hypothesised that below average school performance would be associated with indicators of an unhealthy lifestyle (i.e. low sleep hours, and infrequent exercise participation). Finally, although it was itself used as an outcome, school attendance was additionally examined as a predictor of the other school performance variables. It was hypothesised that below average attendance would be associated with low English and maths attainment, and also with a high occurrence of behavioural sanctions.

\section{MATERIALS AND METHODS}

The data anaylsed here are from the Cornish Academies Project, a large-scale longitudinal investigation of diet, school performance and mental health in secondary school children. The project was carried out by the authors of the current paper in collaboration with three schools in the South West of England. Because the sample and methods used have been discussed extensively in previous publications (see Richards, Malthouse \& Smith [14]; Richards \& Smith [15]) they will only be briefly recapped here.

\subsection{Participants}

Three thousand and seventy one secondary school children from three schools in the South West of England were asked to participate. Two thousand six hundred and ten agreed, 2030 completed the questionnaires at the first timepoint (T1), and 2307 completed them at the second (T2); the two cross-sections of data were collected six months apart. At both time-points the sample was comprised of similar numbers of males and females, and of children from each year group of secondary school education. However, those who responded to the questionnaires were found to differ on a number of aspects of demography from those who did not (for analyses of the representativeness of the samples from $\mathrm{T} 1$ and $\mathrm{T} 2$, see Richards,
Malthouse \& Smith [14], and Richards \& Smith [15], respectively).

\subsection{Materials}

Participants were administered the 29-item Diet and Behaviour Scale [14], as well as single-items relating to general health, stress, anxiety, depression, sleep, and exercise participation. However, only the latter two of these are of importance to the current paper. Average nightly sleep duration was assessed via the following question: "How long do you sleep for each night?" Participants gave their answers in number of hours. They were then asked: "How often do you take part in sports or other types of physical exercise?" Participation in mild, moderate, and vigorous exercise were each recorded on a four-point scale $(1=$ three times a week or more, 2 = once or twice a week, 3 = about once to three times a month, $4=$ never/hardly ever). These three items were factor analysed into a singlefactor solution, the details of which have been reported by Richards, Malthouse, \& Smith [14].

\subsection{Design and Procedure}

Schoolteachers administered questionnaires to the students at their schools at T1 and T2. Information relating to demography (sex, age, school attended, school year, presence/absence of a SEN status, and eligibility/ineligibility to receive FSM) and school performance (attendance, English and maths attainment at $\mathrm{KS} 3 / \mathrm{KS} 4$, and number of detentions/behavioural points incurred throughout term time) was subsequently acquired through the School Information Management System at both timepoints. These data were then merged into a single anonymous database.

\subsection{Statistical Methods}

As different systems for grading and behavioural sanctions were present across the schools and Key Stages, each of these outcome variables was dichotomised. The outcome of school attendance was also dichotomised, in this case using a median split, because the data were considerably skewed. Relationships between school performance and demographic/lifestyle factors were initially explored at the univariate level using Chi-square and Chi-square tests for linear association when the predictor variables were categorical, and with between-subjects t- 
tests when they were continuous. These analyses were then followed-up with binary logistic regression (using enter method) to help determine whether the relationships observed were independent. As the two cross-sections were known to vary somewhat, both in terms of size and demography (e.g. the sample at T2 contained a larger proportion of children with a SEN status), separate analyses were conducted at both time-points.

\section{RESULTS}

Considerable variance was observed in relation to school attendance, attainment, and the occurrence of behavioural sanctions in both cross-sections of data. Descriptive statistics and frequencies relating to these variables at $\mathrm{T} 1$ and $\mathrm{T} 2$, as a whole, and within each of the three schools from which the data came, are shown in Table 1.

\subsection{Dichotomisation of School Perfor- mance Outcomes}

Due to the data being heavily skewed, school attendance was dichotomised via a median split. The medians observed were $95.59 \%$ at $\mathrm{T} 1$ and $93.4 \%$ at T2, which are close to the minimum of $95 \%$ recommended by the UK government. This is also considered a useful cut-off point, as $73 \%$ of students who achieve $\geq 95 \%$ accomplish five or more GCSEs at grades $A^{*}$ to $C$ [2]. However, it was decided that a median split would be more suitable than splitting the distribution into those who achieved $95 \%$ attendance or more and those who did not. This is because although the distribution of high and low attenders determined through this method would be relatively balanced at T1 (high $=55.3 \%$, low $=44.7 \%$ ), this would not be the case at T2 (high $=36.9 \%$, low $=63.1 \%$ ).

English and maths attainment could not be dichotomised using such a simple method as that utilised for attendance. This was because the grading systems differed between KS3 and KS4, and also between academies. At KS3, each of the academies utilised a system ranging from $8 \mathrm{a}$ (highest) to 1c (lowest), with three discreet categories within each grade boundary (e.g. 8a, $8 b, 8 c)$. This gave 24 potential grade categories. At KS4, however, each academy used a different system for grading work. Academy 1 used a system ranging from $A+$ to $G$-, in which three separate distinctions were obtainable within each grade boundary from A-G (e.g. A+, A, A-). A ' $U$ ' was also available in this system, indicating an ungraded standard of work (i.e. a fail grade), thereby meaning that 22 discreet grade categories were present. Academy 2 used a system that ranged from $A^{*}$ to $G$ (with $U$ again indicating that work was of an ungraded standard). In this case, however, no further differentiation within grade boundaries was made, resulting in only nine separate categories. Academy 3 used a system ranging from $A^{*} a$ (highest) to $\mathrm{Gc}$ (lowest), with a $U$ indicating ungraded work. Each grade boundary (from A-G) was differentiated into three distinct levels (e.g. Aa, Ab, Ac), providing 25 possible grades.

Due to the array of separate grading methods used by the three academies, the data needed to be recoded before being analysed as a whole. For each system, grades were ranked from highest to lowest, and then recoded via median split to provide a high attainment group and a low attainment group (the group to which each child was assigned being based on whether they were above or below the median at KS3/KS4 within the academy that they attended). Composite variables consisting of $\mathrm{KS} 3$ and $\mathrm{KS} 4$ for the whole sample were then created for both English and maths.

As with attainment, the method used for recording behavioural sanctions also differed between schools. Academies 1 and 2 provided exact numbers of detentions received by students over the course of the school year, whereas Academy 3 utilised a behavioural points system (higher numbers indicating more occurrences of problem behaviour). Therefore, in order to be able to analyse the sample as a whole, a compound dichotomous variable was created consisting of a 'good behaviour' group and a 'bad behaviour' group. The behavioural points variable provided by Academy 3 was split into quintiles, with those in the lowest $80 \%$ being placed into the good behaviour group, along with those from Academies 1 and 2 who did not receive any detentions. The bad behaviour group was comprised of those from Academy 3 who acquired the highest $20 \%$ of behavioural points, and those from Academies 1 and 2 who received one detention or more. Recoding into quintiles was determined to be a good method of categorising those from Academy 3 as it allowed for similar percentages of students from all schools to be placed into each of the two behaviour groups. 
Table 1. Descriptive statistics and frequencies for school performance variables at T1 and T2

\begin{tabular}{|c|c|c|c|c|c|c|c|c|c|c|c|c|}
\hline & & & \multicolumn{2}{|c|}{$N$} & \multicolumn{2}{|c|}{ Min } & \multicolumn{2}{|c|}{ Max } & \multicolumn{2}{|c|}{$M$} & \multicolumn{2}{|c|}{$S D$} \\
\hline & & & T1 & T2 & T1 & T2 & T1 & T2 & T1 & T2 & T1 & T2 \\
\hline \multirow[t]{4}{*}{ School attendance } & & Total sample & 3040 & 3019 & $0 \%$ & $0 \%$ & $100 \%$ & $100 \%$ & $93.08 \%$ & $90.73 \%$ & 9.66 & 10.46 \\
\hline & & Academy 1 & 948 & 928 & $0 \%$ & $0 \%$ & $100 \%$ & $99.13 \%$ & $94.78 \%$ & $88.35 \%$ & 7.49 & 11.95 \\
\hline & & Academy 2 & 1346 & 1336 & $0 \%$ & $0 \%$ & $98.5 \%$ & $99.37 \%$ & $91.83 \%$ & $91.46 \%$ & 10.19 & 9.67 \\
\hline & & Academy 3 & 746 & 755 & $0 \%$ & $0 \%$ & $100 \%$ & $100 \%$ & $93.16 \%$ & $92.38 \%$ & 10.73 & 9.27 \\
\hline \multirow{6}{*}{$\begin{array}{l}\text { English } \\
\text { attainment }\end{array}$} & KS3 & Academy 1 & 364 & 400 & 9 & 7 & 23 & 22 & 13.88 & 12.39 & 2.32 & 2.45 \\
\hline & & Academy 2 & 1049 & 799 & 6 & 1 & 24 & 20 & 11.63 & 9.98 & 2.97 & 2.88 \\
\hline & & Academy 3 & 395 & 408 & 4 & 4 & 21 & 18 & 12.43 & 10.61 & 2.66 & 2.86 \\
\hline & KS4 & Academy 1 & 552 & 558 & 1 & 1 & 22 & 22 & 11.17 & 9.92 & 4.2 & 4.12 \\
\hline & & Academy 2 & 259 & 524 & 1 & 1 & 8 & 8 & 3.34 & 3.51 & 1.09 & 1.18 \\
\hline & & Academy 3 & 322 & 336 & 6 & 1 & 25 & 25 & 16.49 & 10.6 & 4.99 & 3.74 \\
\hline \multirow{6}{*}{$\begin{array}{l}\text { Maths } \\
\text { attainment }\end{array}$} & KS3 & Academy 1 & 373 & 401 & 4 & 2 & 24 & 21 & 12.53 & 10.71 & 3.12 & 3.57 \\
\hline & & Academy 2 & 534 & 802 & 2 & 1 & 22 & 24 & 10.59 & 9.89 & 3.45 & 3.78 \\
\hline & & Academy 3 & 391 & 409 & 1 & 1 & 21 & 18 & 11.65 & 10.25 & 3.77 & 3.98 \\
\hline & KS4 & Academy 1 & 551 & 556 & 1 & 1 & 22 & 22 & 13.1 & 10.25 & 4.99 & 5.06 \\
\hline & & Academy 2 & 780 & 524 & 1 & 1 & 9 & 8 & 4.17 & 3.96 & 1.68 & 1.44 \\
\hline & & Academy 3 & 331 & 324 & 4 & 1 & 25 & 24 & 11.27 & 12.62 & 3.6 & 4.81 \\
\hline \multirow{3}{*}{$\begin{array}{l}\text { Behavioural } \\
\text { sanctions }\end{array}$} & Detentions & Academy 1 & 954 & 938 & 0 & 0 & 37 & 38 & 0.62 & 0.66 & 2.16 & 2.24 \\
\hline & Detentions & Academy 2 & 1346 & 1336 & 0 & 0 & 6 & 17 & 0.14 & 0.69 & 0.55 & 1.89 \\
\hline & Points & Academy 3 & 740 & 926 & 0 & 0 & 135 & 166 & 6.82 & 7 & 15.27 & 17.66 \\
\hline
\end{tabular}

Note. The grading systems for English and maths attainment varied between academies and Key Stages. At Key Stage 3, each academy graded students from 8 a (highest) to $1 \mathrm{c}$ (lowest), providing a possible range of 1 to 24 . At Key Stage 4 , Al: A+ (highest) to G-, with U (lowest) indicating ungraded work (possible range =1-22); Academy 2: A* (highest) to G, with a U (lowest) indicating ungraded work Scores provided for behavioural sanctions from Academies 1 and 2 relate to the number of detentions incurred, whereas those for Academy 3 relate to the number of 'behavioural points' accumulated throughout term-time. In each case, high 
Table 2. Chi-square values and cross-tabulations between school performance outcomes and categorical demographic/lifestyle variables at T1

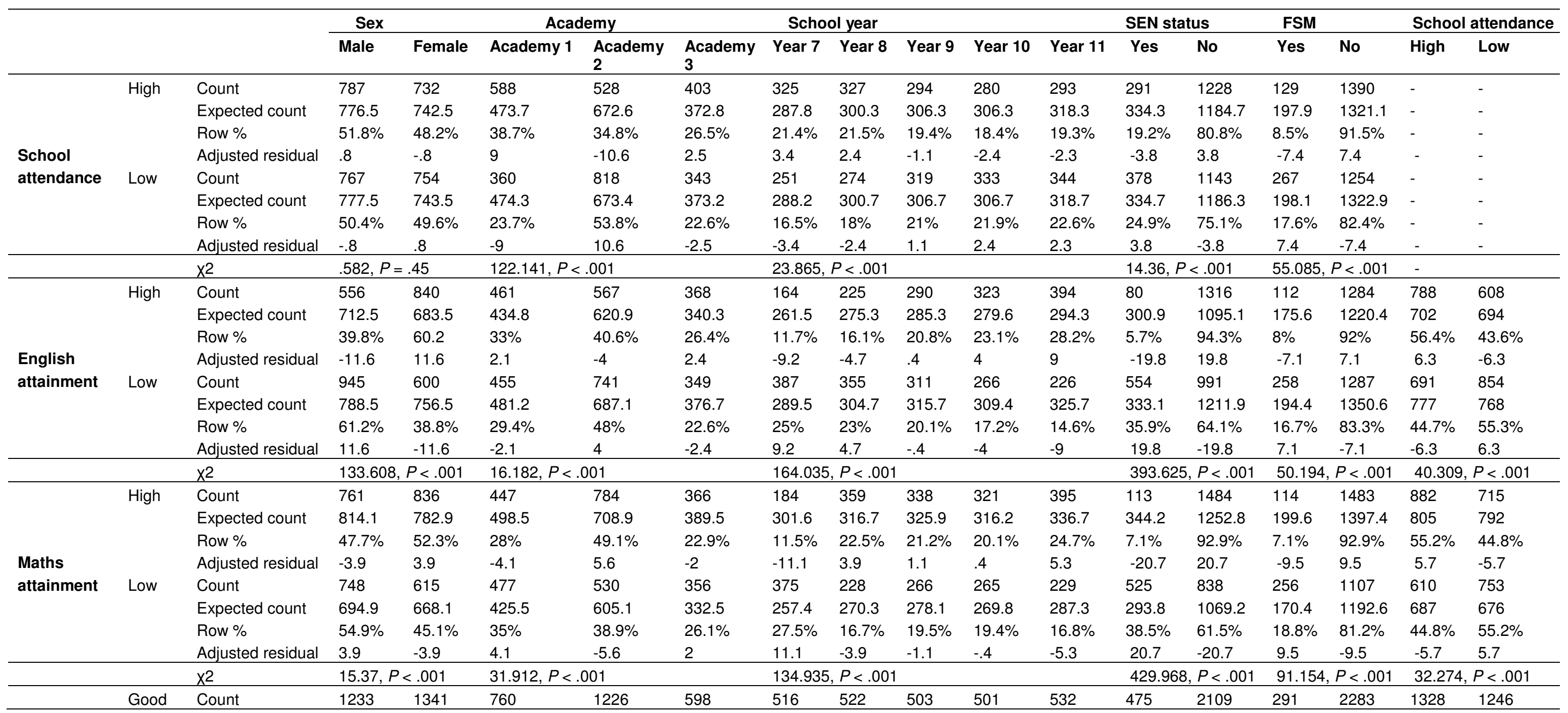




\begin{tabular}{|c|c|c|c|c|c|c|c|c|c|c|c|c|c|c|c|c|c|c|}
\hline & & & \multicolumn{2}{|l|}{ Sex } & \multicolumn{2}{|c|}{ Academy } & \multicolumn{4}{|c|}{ School year } & \multirow[b]{2}{*}{ Year 10} & \multirow[b]{2}{*}{ Year 11} & \multicolumn{2}{|c|}{ SEN status } & \multicolumn{2}{|l|}{ FSM } & \multicolumn{2}{|c|}{ School attendance } \\
\hline & & & Male & Female & Academy 1 & $\begin{array}{l}\text { Academy } \\
2\end{array}$ & $\begin{array}{l}\text { Academy } \\
3\end{array}$ & Year 7 & Year 8 & Year 9 & & & Yes & No & Yes & No & High & Low \\
\hline \multirow{8}{*}{$\begin{array}{l}\text { Behavioural } \\
\text { sanctions }\end{array}$} & \multirow{8}{*}{$\mathrm{Bad}$} & Expected count & 1313.4 & 1260.6 & 810.9 & 1144.1 & 629 & 484.5 & 509.2 & 519.4 & 519.4 & 541.5 & 565.4 & 2018.6 & 334.1 & 2239.9 & 1289.6 & 1284.4 \\
\hline & & Row \% & $47.9 \%$ & $52.1 \%$ & $29.4 \%$ & $47.4 \%$ & $23.1 \%$ & $20 \%$ & $20.3 \%$ & $19.5 \%$ & $19.5 \%$ & $20.7 \%$ & $18.4 \%$ & $81.6 \%$ & $11.3 \%$ & $88.7 \%$ & $51.6 \%$ & $48.4 \%$ \\
\hline & & Adjusted residual & -8.2 & 8.2 & -5.6 & 8.4 & -3.7 & 4.1 & 1.6 & -2.1 & -2.3 & -1.2 & -11.1 & 11.1 & -6.5 & 6.5 & 3.9 & -3.9 \\
\hline & & Count & 312 & 142 & 194 & 120 & 142 & 54 & 77 & 108 & 110 & 105 & 190 & 265 & 102 & 352 & 189 & 265 \\
\hline & & Expected count & 231.6 & 222.4 & 143.1 & 201.9 & 111 & 85.5 & 89.8 & 91.6 & 91.6 & 95.5 & 99.6 & 355.4 & 58.9 & 395.1 & 227.4 & 226.6 \\
\hline & & Row \% & $68.7 \%$ & $31.3 \%$ & $42.5 \%$ & $26.3 \%$ & $31.1 \%$ & $11.9 \%$ & $17 \%$ & $23.8 \%$ & $24.2 \%$ & $23.1 \%$ & $41.8 \%$ & $58.2 \%$ & $22.5 \%$ & $77.5 \%$ & $41.6 \%$ & $58.4 \%$ \\
\hline & & Adjusted residual & 8.2 & -8.2 & 5.6 & -8.4 & 3.7 & -4.1 & -1.6 & 2.1 & 2.3 & 1.2 & 11.1 & -11.1 & 6.5 & -6.5 & -3.9 & 3.9 \\
\hline & & $x^{2}$ & 66.946 , & $<<.001$ & $70.571, P<$. & 001 & & 24.678, & $P<.001$ & & & & 123.67 & $P<.001$ & $42.57 \mathrm{f}$ & $<<.001$ & 15.323 & $P<.001$ \\
\hline
\end{tabular}

Table 3. Chi-square values and cross-tabulations between school performance outcomes and categorical demographic/lifestyle variables at T2

\begin{tabular}{|c|c|c|c|c|c|c|c|c|c|c|c|c|c|c|c|c|c|c|}
\hline & & & \multicolumn{2}{|c|}{ Sex } & \multicolumn{3}{|c|}{ Academy } & \multicolumn{5}{|c|}{ School year } & \multicolumn{2}{|c|}{ SEN status } & \multicolumn{2}{|c|}{ FSM } & \multicolumn{2}{|c|}{ School attendance } \\
\hline & & & Male & Female & Academy 1 & Academy 2 & Academy 3 & Year 7 & Year 8 & Year 9 & Year 10 & Year 11 & Yes & No & Yes & No & High & Low \\
\hline & High & Count & 546 & 558 & 324 & 727 & 452 & 365 & 324 & 344 & 302 & 165 & 404 & 1098 & 130 & 1370 & - & - \\
\hline & & Expected count & 538.8 & 565.2 & 462 & 665.1 & 375.9 & 281.2 & 295.6 & 304.6 & 303.1 & 315.6 & 440.8 & 1061.2 & 190.9 & 1309.1 & - & - \\
\hline & & Row \% & $49.5 \%$ & $50.5 \%$ & $21.6 \%$ & $48.4 \%$ & $30.1 \%$ & $24.3 \%$ & $21.6 \%$ & $22.9 \%$ & $20.1 \%$ & $11 \%$ & $26.9 \%$ & $73.1 \%$ & $8.7 \%$ & $91.3 \%$ & - & - \\
\hline \multirow{9}{*}{$\begin{array}{l}\text { School } \\
\text { attendance }\end{array}$} & & Adjusted residual & .6 & -.6 & -10.9 & 4.5 & 6.4 & 7.8 & 2.6 & 3.6 & -.1 & -13.5 & -2.9 & 2.9 & -6.7 & 6.7 & - & - \\
\hline & Low & Count & 455 & 492 & 604 & 609 & 303 & 199 & 269 & 267 & 306 & 468 & 480 & 1030 & 253 & 1256 & - & - \\
\hline & & Expected count & 462.2 & 484.8 & 466 & 670.9 & 379.1 & 282.8 & 297.4 & 306.4 & 304.9 & 317.4 & 443.2 & 1066.8 & 192.1 & 1316.9 & - & - \\
\hline & & Row \% & $48 \%$ & $52 \%$ & $39.8 \%$ & $40.2 \%$ & $20 \%$ & $13.2 \%$ & $17.8 \%$ & $17.7 \%$ & $20.3 \%$ & $31 \%$ & $31.8 \%$ & $68.2 \%$ & $16.8 \%$ & $83.2 \%$ & - & - \\
\hline & & Adjusted residual & -.6 & .6 & 10.9 & -4.5 & -6.4 & -7.8 & -2.6 & -3.6 & .1 & 13.5 & 2.9 & -2.9 & 6.7 & -6.7 & - & - \\
\hline & & $x^{2}$ & \multicolumn{2}{|c|}{$.406, P=.52$} & \multicolumn{3}{|c|}{$124.257, P<.001$} & \multicolumn{5}{|c|}{$208.702, P<.001$} & \multicolumn{2}{|c|}{$8.686, P=.003$} & \multicolumn{2}{|c|}{$44.424, P<.001$} & - & \\
\hline & High & Count & 413 & 593 & 474 & 590 & 366 & 161 & 280 & 339 & 292 & 346 & 208 & 1212 & 121 & 1297 & 757 & 663 \\
\hline & & Expected count & 491.4 & 514.6 & 443.2 & 631.4 & 355.4 & 264.2 & 280.1 & 290.1 & 286.8 & 296.8 & 411 & 1009 & 177 & 1241 & 711 & 709 \\
\hline & & Row \% & $41.1 \%$ & $58.9 \%$ & $33.1 \%$ & $41.3 \%$ & $25.6 \%$ & $11.4 \%$ & $19.7 \%$ & $23.9 \%$ & $20.6 \%$ & $24.4 \%$ & $14.6 \%$ & $85.4 \%$ & $8.5 \%$ & $91.5 \%$ & $53.3 \%$ & $46.7 \%$ \\
\hline English & & Adjusted residual & -7 & 7 & 2.4 & -3.1 & .9 & -9.8 & .0 & 4.5 & .5 & 4.4 & -16.5 & 16.5 & -6.2 & 6.2 & 3.4 & -3.4 \\
\hline \multirow[t]{5}{*}{ Attainment } & Low & Count & 582 & 449 & 454 & 732 & 378 & 390 & 304 & 266 & 306 & 273 & 649 & 892 & 248 & 1291 & 728 & 818 \\
\hline & & Expected count & 503.6 & 527.4 & 484.8 & 690.6 & 388.6 & 286.8 & 303.9 & 314.9 & 311.2 & 322.2 & 446 & 1095 & 192 & 1347 & 774 & 772 \\
\hline & & Row \% & $56.5 \%$ & $43.5 \%$ & $29 \%$ & $46.8 \%$ & $24.2 \%$ & $25.3 \%$ & $19.8 \%$ & $17.3 \%$ & $19.9 \%$ & $17.7 \%$ & $42.1 \%$ & $57.9 \%$ & $16.1 \%$ & $83.9 \%$ & $47.1 \%$ & $52.9 \%$ \\
\hline & & Adjusted residual & 7 & -7 & -2.4 & 3.1 & -.9 & 9.8 & .0 & -4.5 & -.5 & -4.4 & 16.5 & -16.5 & 6.2 & -6.2 & -3.4 & 3.4 \\
\hline & & $x^{2}$ & \multicolumn{2}{|c|}{$48.305, P<.001$} & \multicolumn{3}{|c|}{$9.9, P=.007$} & \multicolumn{5}{|c|}{$109.137, P<.001$} & \multicolumn{2}{|c|}{$271.11, P<.001$} & \multicolumn{2}{|c|}{$38.838, P<.001$} & \multicolumn{2}{|c|}{$11.456, P=.001$} \\
\hline
\end{tabular}




\begin{tabular}{|c|c|c|c|c|c|c|c|c|c|c|c|c|c|c|c|c|c|c|}
\hline & & & \multicolumn{2}{|c|}{ Sex } & \multicolumn{3}{|c|}{ Academy } & \multicolumn{5}{|c|}{ School year } & \multicolumn{2}{|c|}{ SEN status } & \multicolumn{2}{|c|}{ FSM } & \multicolumn{2}{|c|}{ School attendance } \\
\hline & & & Male & Female & Academy 1 & Academy 2 & Academy 3 & Year 7 & Year 8 & Year 9 & Year 10 & Year 11 & Yes & No & Yes & No & High & Low \\
\hline \multirow{9}{*}{$\begin{array}{l}\text { Maths } \\
\text { attainment }\end{array}$} & High & Count & 476 & 512 & 456 & 562 & 364 & 193 & 314 & 341 & 185 & 333 & 195 & 1173 & 110 & 1256 & 749 & 622 \\
\hline & & Expected count & 482.1 & 505.9 & 429.2 & 613.5 & 339.4 & 255.9 & 271.2 & 279.5 & 273 & 286.4 & 393.4 & 974.6 & 170.8 & 1195.3 & 685.7 & 685.3 \\
\hline & & Row \% & $48.2 \%$ & $51.8 \%$ & $33 \%$ & $40.7 \%$ & $26.3 \%$ & $14.1 \%$ & $23 \%$ & $25 \%$ & $13.5 \%$ & $24.4 \%$ & $14.3 \%$ & $85.7 \%$ & $8.1 \%$ & $91.9 \%$ & $54.6 \%$ & $45.4 \%$ \\
\hline & & Adjusted residual & -.5 & .5 & 2.1 & -3.8 & 2.1 & -6 & 4 & 5.6 & -8.1 & 4.2 & -16.2 & 16.2 & -6.8 & 6.8 & 4.7 & -4.7 \\
\hline & Low & Count & 514 & 527 & 471 & 763 & 369 & 360 & 272 & 263 & 405 & 286 & 655 & 933 & 259 & 1327 & 732 & 858 \\
\hline & & Expected count & 507.9 & 533.1 & 497.8 & 711.5 & 393.6 & 297.1 & 314.8 & 324.5 & 317 & 332.6 & 456.6 & 1131.4 & 198.3 & 1387.8 & 795.3 & 794.7 \\
\hline & & Row \% & $49.4 \%$ & $50.6 \%$ & $29.4 \%$ & $47.6 \%$ & $23 \%$ & $22.7 \%$ & $17.2 \%$ & $16.6 \%$ & $25.5 \%$ & $18 \%$ & $41.2 \%$ & $58.8 \%$ & $16.3 \%$ & $83.7 \%$ & $46 \%$ & $54 \%$ \\
\hline & & Adjusted residual & .5 & -.5 & -2.1 & 3.8 & -2.1 & 6 & -4 & -5.6 & 8.1 & -4.2 & 16.2 & -16.2 & 6.8 & -6.8 & -4.7 & 4.7 \\
\hline & & $x^{2}$ & \multicolumn{2}{|c|}{$.291, P=.59$} & \multicolumn{3}{|c|}{$14.485, P=.001$} & \multicolumn{5}{|c|}{$133.463, P<.001$} & \multicolumn{2}{|c|}{$261.366, P<.001$} & \multicolumn{2}{|c|}{$45.977, P<.001$} & \multicolumn{2}{|c|}{$21.749, P<.001$} \\
\hline \multirow{9}{*}{$\begin{array}{l}\text { Behavioural } \\
\text { sanctions }\end{array}$} & Good & Count & 720 & 900 & 740 & 1022 & 746 & 468 & 476 & 457 & 433 & 466 & 584 & 1720 & 249 & 2051 & 1202 & 1102 \\
\hline & & Expected count & 791.5 & 828.5 & 735.9 & 1046.3 & 725.8 & 430.4 & 454.3 & 467.4 & 464.3 & 483.6 & 674 & 1630 & 293.4 & 2006.6 & 1149.7 & 1154.3 \\
\hline & & Row \% & $44.4 \%$ & $55.6 \%$ & $29.5 \%$ & $40.7 \%$ & $29.7 \%$ & $20.3 \%$ & $20.7 \%$ & $19.9 \%$ & $18.8 \%$ & $20.3 \%$ & $25.3 \%$ & $74.7 \%$ & $10.8 \%$ & $89.2 \%$ & $52.2 \%$ & $47.8 \%$ \\
\hline & & Adjusted residual & -7.7 & 7.7 & .4 & -2.1 & 1.9 & 4.2 & 2.4 & -1.1 & -3.4 & -1.9 & -8.6 & 8.6 & -5.8 & 5.8 & 4.6 & -4.6 \\
\hline & $\mathrm{Bad}$ & Count & 283 & 150 & 199 & 313 & 180 & 91 & 114 & 150 & 170 & 162 & 291 & 396 & 132 & 555 & 289 & 395 \\
\hline & & Expected count & 211.5 & 221.5 & 203.1 & 288.7 & 200.2 & 128.6 & 135.7 & 139.6 & 138.7 & 144.4 & 201 & 486 & 87.6 & 599.4 & 341.3 & 342.7 \\
\hline & & Row \% & $65.4 \%$ & $34.6 \%$ & $28.8 \%$ & $45.2 \%$ & $26 \%$ & $13.2 \%$ & $16.6 \%$ & $21.8 \%$ & $24.7 \%$ & $23.6 \%$ & $42.4 \%$ & $57.6 \%$ & $19.2 \%$ & $80.8 \%$ & $42.3 \%$ & $57.7 \%$ \\
\hline & & Adjusted residual & 7.7 & -7.7 & -.4 & 2.1 & -1.9 & -4.2 & -2.4 & 1.1 & 3.4 & 1.9 & 8.6 & -8.6 & 5.8 & -5.8 & -4.6 & 4.6 \\
\hline & & $x^{2}$ & \multicolumn{2}{|c|}{$59.808, P<.001$} & \multicolumn{3}{|c|}{$5.327, P=.07$} & \multicolumn{5}{|c|}{$31.721, P<.001$} & \multicolumn{2}{|c|}{$73.992, P<.001$} & \multicolumn{2}{|c|}{$33.445, P<.001$} & \multicolumn{2}{|c|}{$20.755, P<.001$} \\
\hline
\end{tabular}




\subsection{Categorical Correlates of School Performance}

Chi-square tests were used to examine associations between school performance and the categorical demographic/lifestyle variables. Cross-tabulations, $X 2$, and $P$ values for these analyses from $\mathrm{T} 1$ and $\mathrm{T} 2$ are displayed in Tables 2 and 3 , respectively.

High attainment and good behaviour were more common in females than males, though no effects were observed regarding maths attainment at T2, or school attendance at either time-point. Differences between the schools were detected for each of the outcomes at both timepoints, although the association with behavioural sanctions at T2 was only marginally significant (for the specific differences between academies at $\mathrm{T} 1$ and $\mathrm{T} 2$, see the cross-tabulations presented in Tables 2 and 3, respectively). The year group attended was also associated with each of the school performance outcomes at both time-points. Chi-square tests for linear association showed that school attendance was negatively associated with year group, at both $\mathrm{T} 1, \mathrm{X} 2(1, \mathrm{~N}=3040)=20.896, \mathrm{P}<.001$, and T2, $X^{2}(1, N=3009)=165.982, P<.001$. Attainment, on the other hand, was positively associated with school year: English T1, X2 (1, N $=2941)=163.468, \mathrm{P}<.001 ;$ English T2, $\mathrm{X} 2(1, \mathrm{~N}$ $=2957)=65.777, \mathrm{P}<.001 ;$ maths $\mathrm{T} 1, \mathrm{X} 2(1, \mathrm{~N}=$ $2960)=67.421, P<.001 ;$ maths $T 2, X 2(1, N=$ 2952) $=5.324, P=.02$. The occurrence of behavioural sanctions was also found to increase throughout secondary education: $\mathrm{T} 1, \mathrm{X}^{2}(1, \mathrm{~N}=$ $3028)=16.639, \mathrm{P}<.001 ; \mathrm{T} 2, \mathrm{X} 2(1, \mathrm{~N}=2987)=$ 25.36, $\mathrm{P}<.001$. In addition, having a SEN status and being eligible to receive FSM were associated with low school attendance, low English and maths attainment, and high occurrences of behavioural sanctions at both time-points. As would be expected, low school attendance was associated with low English and maths attainment, as well as with a high occurrence of behavioural sanctions at both timepoints.

\subsection{Continuous Correlates of School Performance}

Associations between school performance and continuous lifestyle variables were investigated using between-subjects t-tests. Higher sleep hours and more frequent exercise were associated with the high attendance group at T2, though no such effects were observed at $\mathrm{T} 1$. Strangely, the high English attainment group at
T1 reported lower sleep hours than did the low attainment group, though no such effect was observed at T2. Higher exercise frequency scores were also associated with the high English attainment group, although at both timepoints the effects were only marginally significant. For maths attainment, however, the high performance group achieved significantly higher exercise frequency scores than did the low performance group at both time-points. The high maths attainment group at T2 also reported higher sleep hours, although the effect was only marginally significant, and was not detected at T1. In addition, the good behaviour group at T2 reported higher sleep hours, though no such effect was observed at T1. For $t$ and $P$ values for associations between school performance and continuous demographic/lifestyle variables at both time-points, see Table 4 .

Table 4. Associations between school performance outcomes and continuous demographic/lifestyle variables at T1 and T2

\begin{tabular}{llllll}
\hline & & Sleep & \multicolumn{3}{c}{$\begin{array}{c}\text { Exercise } \\
\text { frequency }\end{array}$} \\
\hline & & $\boldsymbol{t}$ & $\boldsymbol{P}$ & $\boldsymbol{t}$ & $\boldsymbol{P}$ \\
\hline School & $\mathrm{T} 1$ & 1.373 & .17 & 1.385 & .17 \\
attendance & $\mathrm{T} 2$ & 6.66 & $<.001$ & 2.286 & .02 \\
English & $\mathrm{T} 1$ & -2.394 & .02 & 1.877 & .06 \\
attainment & $\mathrm{T} 2$ & -.676 & .5 & 1.675 & .09 \\
Maths & $\mathrm{T} 1$ & -1.404 & .16 & 2.988 & .003 \\
attainment & $\mathrm{T} 2$ & 1.933 & .053 & 3.004 & .003 \\
Behavioural & $\mathrm{T} 1$ & -.841 & .4 & .721 & .47 \\
sanctions & $\mathrm{T} 2$ & 5.782 & $<.001$ & .276 & .78 \\
\hline
\end{tabular}

\subsection{Multivariate Associations between School Performance and Demo- graphic/Lifestyle Variables}

In order to determine whether the demographic and lifestyle factors investigated were independently associated with the school performance outcomes, they were entered simultaneously into binary logistic regression analyses (using enter method). In these analyses female sex, Academy 1, Year 7, absence of a SEN status, ineligibility to receive FSM, and high attendance were set as the comparison groups (this last variable only being entered when attendance was not also the outcome). Sleep (total hours) and exercise (factor score) were entered as continuous variables, with high scores indicating high sleep hours and high exercise frequency. For Wald statistics, odds ratios, 95\% confidence intervals, and $P$ values for the multivariate level analyses of school attendance, English attainment, maths attainment, and behavioural sanctions, see Tables 5, 6, 7, and 8, 
respectively. The current section aims to outline the significant and marginally significant findings, and how they compared to those of the univariate level analyses. However, due to their lack of generalisability to other populations, no discussion of the differences between schools is provided here.

\subsubsection{School attendance}

As with the univariate level analyses, low school attendance remained associated with higher school years, having a SEN status, and being eligible to receive FSM. High sleep hours and exercise frequency also remained associated with high attendance at T2, and a marginally significant relationship between high sleep hours and high attendance at T1 emerged, even though no such effect had been observed at the univariate level. As with the univariate analysis, no association was observed between exercise frequency and school attendance at T1, and no sex differences emerged.

\subsubsection{English attainment}

Low English attainment remained associated with male sex, lower school years, presence of a SEN status, and low school attendance at both time-points. Low scores also remained associated with eligibility for FSM, although at this point the effect at $\mathrm{T} 1$ was only marginally significant. Higher frequency of exercise participation was associated with high English attainment at T1 (an effect that was only marginally significant at the univariate level), though no such effect was detected at T2. Although an association between low sleep hours and high English attainment at T1 was identified at the univariate level, no such effects were observed at either time-point once additional covariates had been controlled for.

\subsubsection{Maths attainment}

Higher school years, SEN status, eligibility to receive $\mathrm{FSM}$, low school attendance, and low exercise frequency all remained significantly associated with low maths attainment at both time-points. Although males were significantly more likely to achieve below average maths attainment at $\mathrm{T} 1$ in the univariate analysis, no sex differences were observed once additional covariates had been controlled for. The marginally significant association between sleep hours and maths attainment at T2 also disappeared during this analysis.

\subsubsection{Behavioural sanctions}

A high occurrence of behavioural sanctions remained associated with male sex, higher school years, SEN status, and eligibility to receive FSM at both time-points. Low school attendance also remained a predictor of a high number of behavioural sanctions at $\mathrm{T} 1$, though the effect at T2 disappeared. The association between low sleep hours and high occurrence of behavioural sanctions at T2 also remained significant, and, as with the univariate analyses, no relationships between behavioural sanctions and exercise frequency were observed.

Table 5. Likelihood of achieving below average school attendance as a function of demographic and lifestyle factors

\begin{tabular}{|c|c|c|c|c|c|c|c|c|}
\hline \multirow{2}{*}{$\begin{array}{l}\text { Predictor } \\
\text { variable }\end{array}$} & \multicolumn{4}{|c|}{ T1 } & \multicolumn{4}{|c|}{ T2 } \\
\hline & Wald & OR & $95 \% \mathrm{Cl}$ & $P$ & Wald & OR & $95 \% \mathrm{Cl}$ & $P$ \\
\hline Sex & .386 & .939 & $.77,1.145$ & .54 & .089 & .97 & $.794,1.185$ & .77 \\
\hline Academy & 102.943 & - & - & $<.001$ & 75.806 & - & - & $<.001$ \\
\hline Academy 2 & 95.228 & 3.038 & $2.43,3.797$ & $<.001$ & 43.83 & .458 & $.364, .577$ & $<.001$ \\
\hline Academy 3 & 3.495 & 1.322 & $.987,1.773$ & .06 & 67.767 & .31 & $.234, .409$ & $<.001$ \\
\hline School year & 15.844 & - & - & .003 & 120.633 & - & - & $<.001$ \\
\hline Year 8 & .233 & 1.084 & $.78,1.507$ & .63 & 7.101 & 1.531 & $1.119,2.093$ & .008 \\
\hline Year 9 & .957 & 1.17 & $.854,1.604$ & .33 & 1.95 & 1.256 & $.912,1.731$ & .16 \\
\hline Year 10 & 2.425 & 1.293 & $.936,1.788$ & .12 & 4.401 & 1.43 & $1.024,1.996$ & .04 \\
\hline Year 11 & 12.502 & 1.793 & $1.297,2.477$ & $<.001$ & 95.011 & 5.576 & $3.947,7.878$ & $<.001$ \\
\hline SEN status & 18.505 & 1.77 & $1.365,2.296$ & $<.001$ & 4.977 & 1.313 & $1.034,1.667$ & .03 \\
\hline FSM & 40.565 & 2.826 & $2.053,3.891$ & $<.001$ & 13.888 & 1.828 & $1.331,2.511$ & $<.001$ \\
\hline Sleep hours & 3.048 & .944 & $.884,1.007$ & .08 & 6.951 & .911 & $.85, .976$ & .008 \\
\hline $\begin{array}{l}\text { Exercise } \\
\text { frequency }\end{array}$ & .625 & .96 & $.869,1.061$ & .43 & 4.027 & .902 & $.816, .998$ & .045 \\
\hline
\end{tabular}


Table 6. Likelihood of achieving below average English attainment as a function of demographic and lifestyle factors

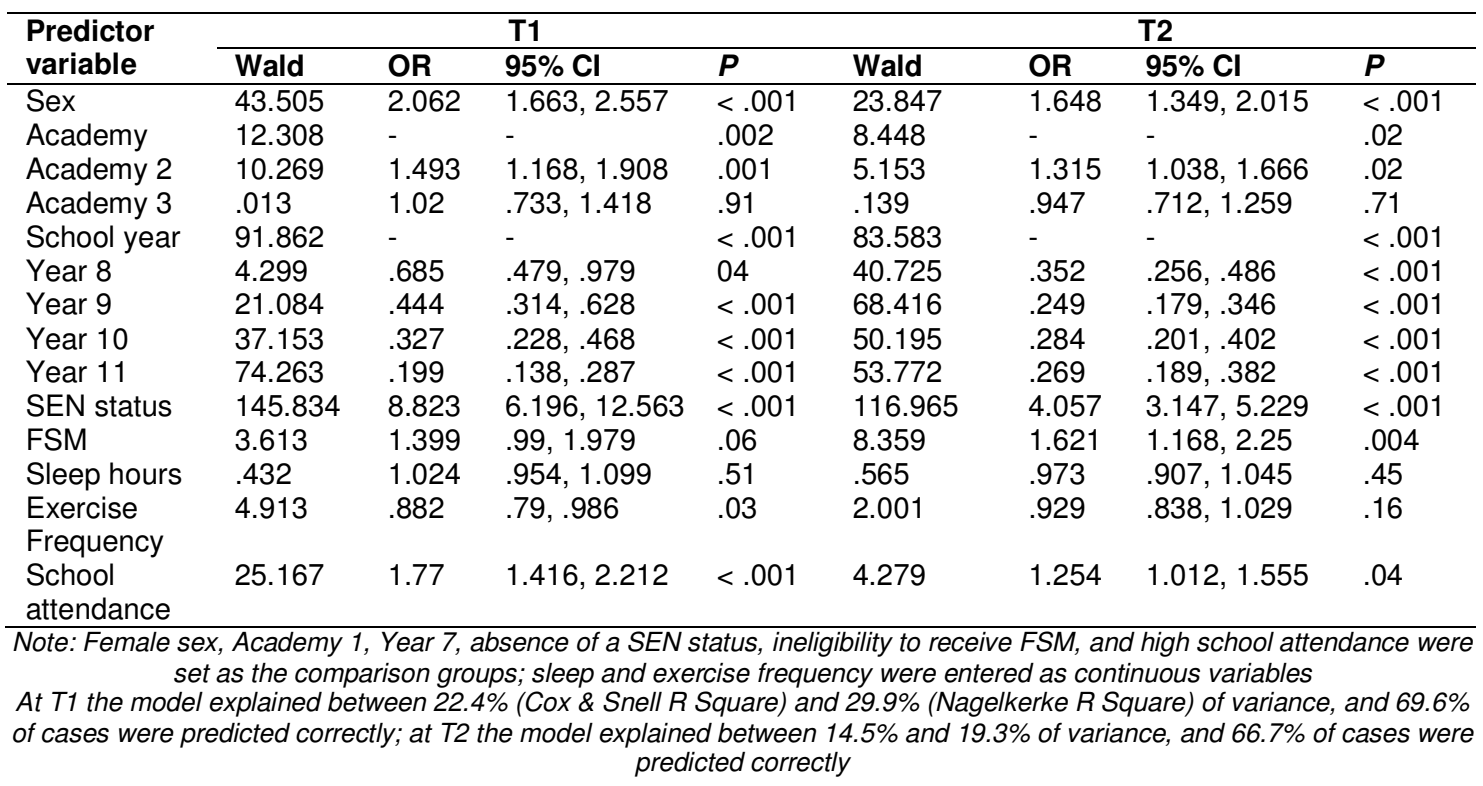

Table 7. Likelihood of achieving below average maths attainment as a function of demographic and lifestyle factors

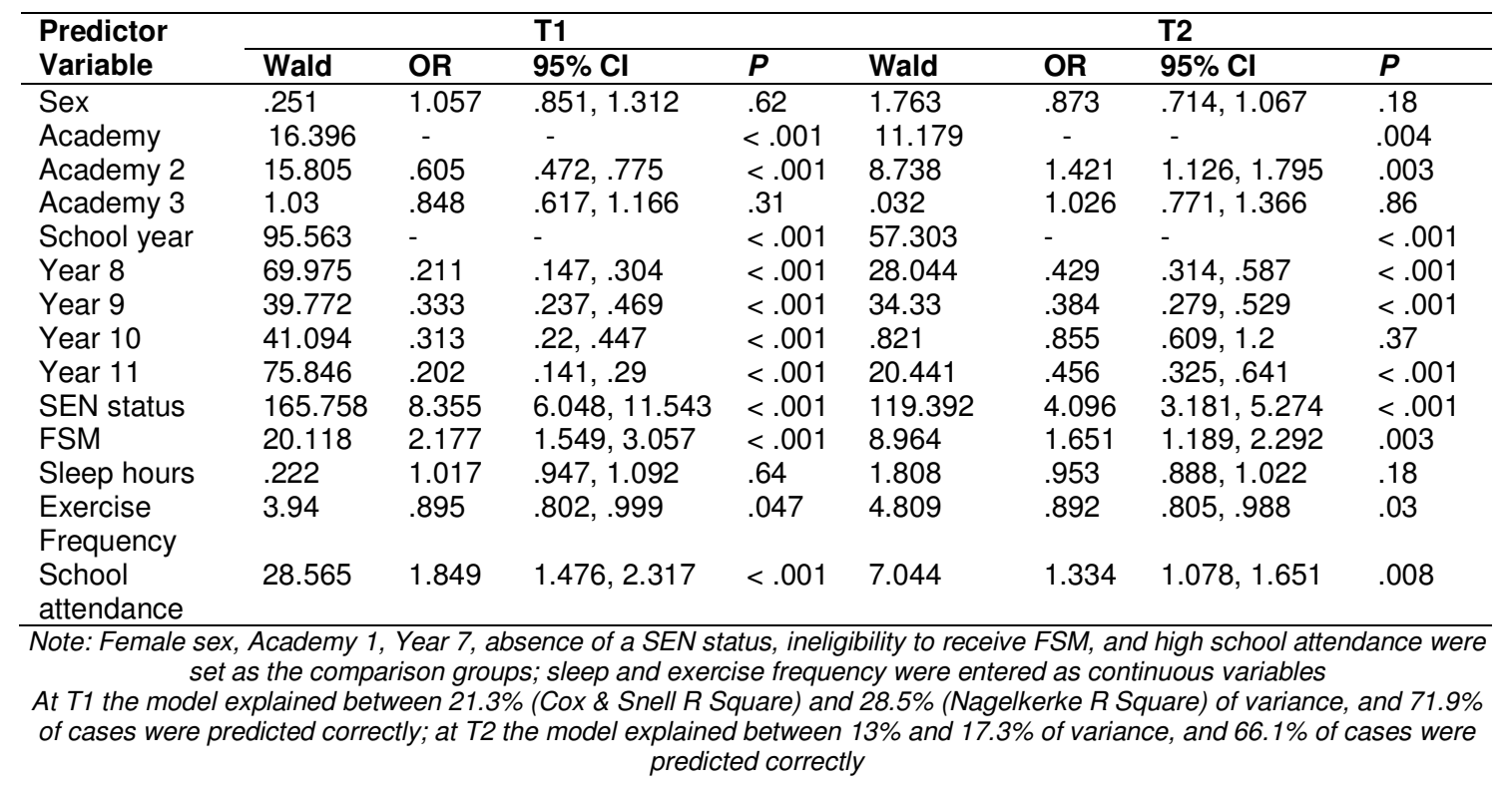

\section{DISCUSSION}

The current paper aimed to examine demographic and lifestyle correlates of school performance in two cross-sections of data from a large-scale longitudinal study of British secondary school children. The hypotheses that low school performance outcomes would be associated with a range of demographic (male sex, school year, eligibility for FSM, presence of SEN) and lifestyle variables (low sleep hours, infrequent exercise participation, low school attendance) were broadly supported. The following two sections aim to discuss those findings relating to demographic and lifestyle variables separately. 
Table 8. Likelihood of achieving a high number of behavioural sanctions as a function of demographic and lifestyle factors

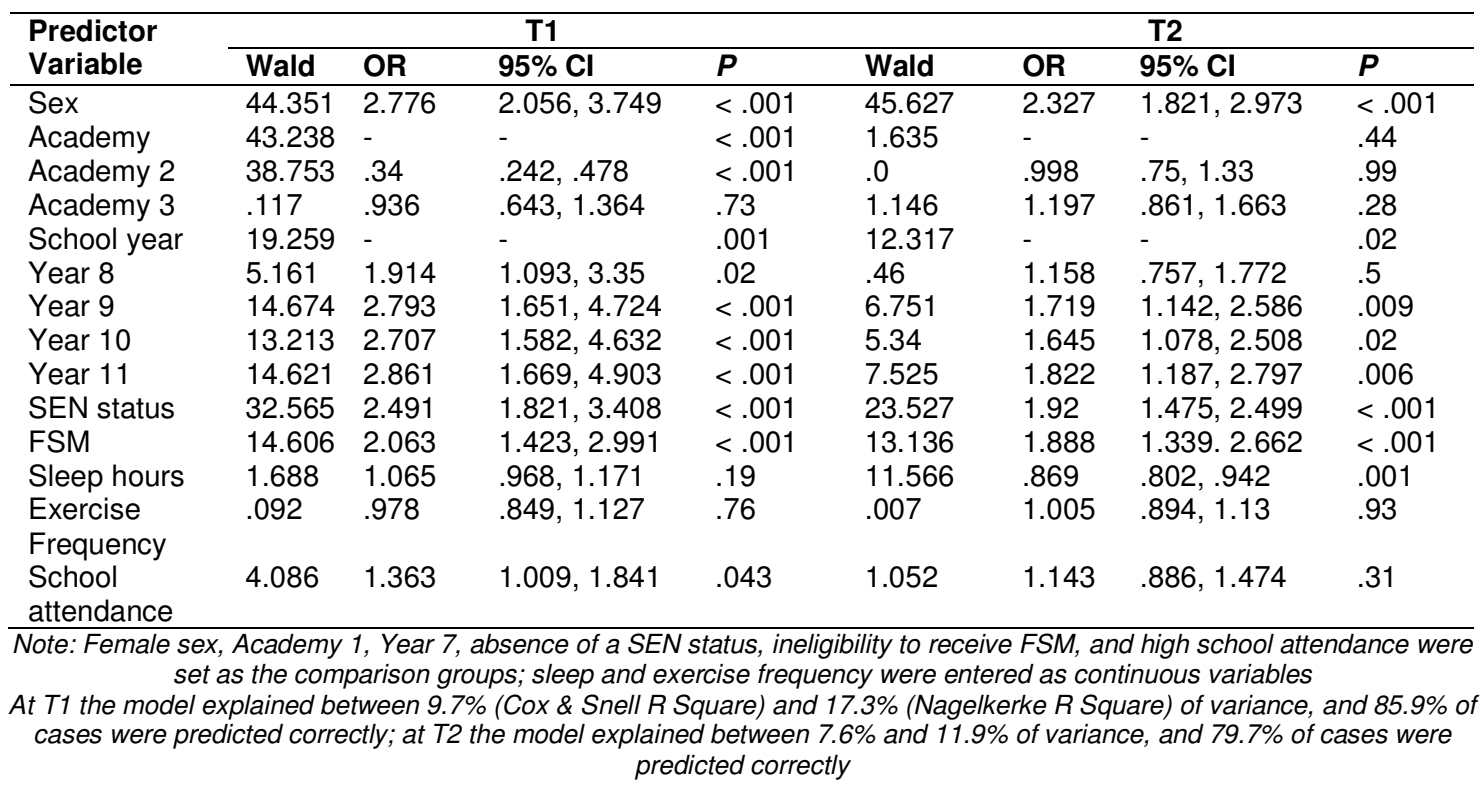

\subsection{Demographic Predictors of School Performance}

Although no sex differences were detected for school attendance, the observation that males achieved lower attainment and incurred a greater number of behavioural sanctions compared to females is similar to previously reported findings (e.g. Gorard, Rees, \& Salisbury [16]). Decreasing attendance and increasing behavioural sanctions throughout secondary school were also observed, findings that are consistent with the observation that antisocial behaviour temporarily increases almost 10-fold during adolescence [17]. However, Moffitt [17] suggests that adolescent delinquency conceals two distinct subgroups of individuals: a large group who are antisocial only during adolescence, and a smaller group that engages in antisocial activities throughout every stage of life. Although this is clearly an important distinction to make if attempting to predict future criminal outcomes, the current methodology would be unable to effectively differentiate between these subgroups. This is an area that may therefore be of particular interest for future research.

As might be expected, presence of a SEN status was consistently associated with low attendance, low attainment, and problem behaviour. Eligibility to receive FSM was also related to each of these outcomes, which is consistent with FSM being an indication of low SES [18], as well as with the observation that low parental SES can predict a child's level of school achievement [19]. As these effects remained significant at the multivariate level, they are also supportive of findings that suggest low income to have an independent effect on school outcomes, even after controlling for key aspects of family background, as well as the child's ability [15].

\subsection{Lifestyle Predictors of School Performance}

Although effects relating to average sleep duration and exercise frequency differed across analyses (i.e. some effects were significant, others were not), they broadly pointed to benefits of high sleep hours and frequent exercise.

At the univariate level, sleep hours were higher in those who achieved high attendance, low behavioural sanctions, and high maths attainment at T2 (although this last effect was only marginally significant). However, no such effects were observed at T1, and a finding in the opposite direction was observed: those who achieved high levels of English attainment at T1 reported lower sleep hours. However, the effect disappeared at the multivariate level, which might imply that the initial observation was a Type 1 error. The positive association between sleep and attendance at T2 did remain significant, and, 
interestingly, a marginally significant effect was also detected at $\mathrm{T1}$, which had not initially appeared at the univariate level. In addition to this, high sleep hours remained associated with good behaviour at T2, though the positive effect relating to maths attainment at T2 disappeared. The findings presented here are congruent with those of a recent meta-analysis [20], which found the school performance of children and adolescents to be positively associated with sleep duration and quality, and negatively associated with sleepiness.

Although no associations were observed with behavioural sanctions, univariate level analyses determined that frequent exercise participation was associated with high attendance at T2, and with high English and maths attainment at both time-points (though those relating to English attainment were both marginally significant). The effects relating to attendance and maths remained significant at the multivariate level; for English attainment, the effect at $\mathrm{T} 1$ became statistically significant, whereas that at T2 disappeared. Overall, the findings discussed here suggest that frequent exercise participation is likely to be beneficial to school performance, which is in line with previous findings (see Singh et al. [21]). However, it should of course be noted that these effects are only cross-sectional, and so cannot be used to infer causation. Furthermore, as frequent exercise participation may be indicative of an overall healthy lifestyle, the effects observed here might potentially be explainable by other factors, such as diet, or personality characteristics.

As well as being an outcome variable, school attendance was investigated as an additional predictor when examining English attainment, maths attainment, and behavioural sanctions. In each case low school attendance was associated with undesirable outcomes, though it should be noted that the effect relating to behavioural sanctions at T2 disappeared at the multivariate level. Taken together, these findings might suggest that children who frequently fail to attend school fall behind in their academic studies (e.g. Taylor [2]), as they essentially only complete the parts of the course for which they are present. Furthermore, such children likely exhibit behavioural problems in the first place given the fact that, as a whole, they appear to incur significantly more behavioural sanctions than do other children, even though they spend less time at school in which to accrue them. Given the other associated demographic risk factors identified in the current sample (e.g. eligibility for FSM), it is likely that such problems stem from the home. These are therefore children that might represent a particularly at-risk subgroup, for which interventions might be beneficial.

\subsection{Limitations}

The current research had a number of strengths. For instance, the samples investigated were large, consisted of children from each year group of secondary education, and were acquired from three different schools. The study also examined several school performance outcomes, used a multivariate approach to analysis, and managed to replicate most of the observed effects across two cross-sections of data. However, some limitations should also be acknowledged. One such issue is that the samples investigated were relatively homogeneous, as the three schools surveyed were all located in a similar geographical area. The generalisability of the findings is therefore somewhat limited. The samples were also not fully representative of the schools that they came from in regards to a number of the demographic variables examined. For instance, males, those with a SEN status, and those eligible for FSM were less likely to complete the questionnaires. Differences in the likelihood of responding were also detected between schools and across year groups (for representativeness of the samples at T1 and T2, see respectively Richards, Malthouse \& Smith [10], and Richards \& Smith [11]).

A further limitation of the current study is that differences in grading and disciplinary systems existed across the three schools surveyed. This meant that data relating to attainment and behavioural sanctions needed to be dichotomised before being analysed as a whole. This process therefore likely decreased the sensitivity of the analyses. However, as this would reduce the probability of detecting statistically significant effects, it also increases the likelihood of those observed being meaningful.

\section{CONCLUSION}

- The results presented here generally support previous findings in the literature.

- A number of independent predictors of low school performance were identified in this sample, such as male sex, presence of a SEN status, eligibility to receive FSM, low 
sleep hours, and infrequent exercise participation.

- Of particular concern was the finding that attendance decreased and behavioural sanctions increased throughout the course of secondary education.

- The identification of covariates of school performance may be useful to future research that takes a multivariate approach to data analysis.

\section{RECOMMENDATIONS}

- $\quad$ Findings from this paper may potentially be useful for identifying at-risk individuals who might benefit from interventions.

- The information provided here could be of use to schoolteachers. For instance, although many will already be aware of certain demographic and lifestyle variables that are related to school performance, some may not be. Improved knowledge might therefore be beneficial for the teachers themselves, as well as for the students that they are teaching.

- Attempts should be made to make sure that no child is at a relative disadvantage to their peers due to differences in demographic background.

- $\quad$ Although the findings relating to sleep and exercise participation are only crosssectional (and so causation cannot be determined), a healthy lifestyle should be promoted regardless. This is because the positive effects associated with a healthy lifestyle are far-reaching, and certainly not limited to school performance.

\section{CONSENT}

Written informed consent was acquired from all participants (as well as from their parents) prior to taking part in the study.

\section{ETHICAL APPROVAL}

Both authors hereby declare that all experiments have been examined and approved by Cardiff University's School of Psychology Ethics Committee (ethical clearance number: EC.12.09.11.3187), and have therefore been performed in accordance with the ethical standards laid down in the 1964 Declaration of Helsinki.

\section{COMPETING INTERESTS}

Authors have declared that no competing interests exist.

\section{REFERENCES}

1. Currie J, Thomas D. Early test scores, socioeconomic status and future outcomes; 1999. Accessed 9 May 2016. Available:http://www.nber.org/papers/w694 3.pdf

DOI: http://dx.doi.org/10.3386/w6943

2. Taylor C. Improving attendance at school. 2012. Accessed 15 March 2016.

Available:www.gov.uk/government/uploads /system/uploads/attachment data/file/1807 72/DFE-00036-

2012 improving attendance at school.pdf

3. Dorling D. Unemployment and health. BMJ. 2009;338(2):b829.

DOI: $10.1136 / \mathrm{bmj} . b 829$.

4. Verbruggen J, Blokland AAJ, van der Geest VR. Effects of employment and unemployment on serious offending in a high-risk sample of men and women from ages 18 to 32 in the Netherlands. $\mathrm{Br} \mathrm{J}$ Criminol. 2012;52(5):845-69.

DOI: http://dx.doi.org/10.1093/bjc/azs023

5. Trades Union Congress. The costs of unemployment. 2010. Accessed 9 May 2016.

Available:https://www.tuc.org.uk/sites/defa ult/files/extras/costsofunemployment.pdf

6. Morris M, Rutt S. Analysis of pupil attendance data in Excellence in Cities (EiC) areas: an interim report. Nottingham: DfES Publications; 2004.

7. Cheeseman Day J, Newburger EC. The big payoff: educational attainment and synthetic estimates of work-life earnings. 2002. Accessed 30 March 2016.

Available:https://www.census.gov/prod/200 2pubs/p23-210.pdf

8. Desforges C, Abouchaar A. The impact of parental involvement, parental support and family education on pupil achievement and adjustment: a literature review. Research report no. 433. Nottingham: DfES; 2003.

9. Farrington DP. Later life outcomes of truants in the Cambridge study. In: Hersov L, Berg I, editors. Unwillingly to school. London: Gaskell; 1996.

10. Reid K. Truancy: short and long-term solutions. London: Routledge; 2002. 
11. Mortimore P, Whitty G. Can school improvement overcome the effects of disadvantage? In: Cox $\mathrm{T}$, editor. Combating educational disadvantage. London: Falmer; 2000.

12. Hinshaw SP. Externalizing behavior problems and academic underachievement in childhood and adolescence: casual relationships and underlying mechanisms. Psychol Bull. 1992;111(1):127-55.

DOI: 10.1037/0033-2909.111.1.127.

13. Pajer KA. What happens to "bad" girls: A review of the adult outcomes of antisocial adolescent girls. Am J Psychiatry. 1998;155(7):862-70.

DOI: 10.1176/ajp.155.7.862.

14. Richards G, Malthouse A, Smith AP. The diet and behaviour scale (DABS): testing a new measure of food and drink consumption in a cohort of secondary school children from the south west of England. J food res. 2015;4(3):148-61.

DOI: $10.5539 /$ jfr.v4n3p148

15. Richards G, Smith AP. Caffeine consumption and self-assessed stress, anxiety, and depression in secondary school children. J Psychopharmacol. 2015;29(12):1236-47.

DOI: $10.1177 / 0269881115612404$.

16. Gorard S, Rees G, Salisbury J. Investigating the patterns of differential attainment of boys and girls at school. $\mathrm{Br}$ Educ Res J. 2001;27(2):125-39.

DOI: $10.1080 / 01411920120037090$.
17. Moffitt TE. Adolescence-limited and lifecourse-persistent antisocial behavior: A developmental taxonomy. Psychol Rev. 1993;100(4):674-701.

DOI: $10.1037 / 0033-295 X .100 .4 .674$.

18. Shuttleworth I. The relationship between social deprivation, as measured by individual free school meal eligibility, and educational attainment at GCSE in Northern Ireland: A preliminary investigation. $\mathrm{Br}$ Educ Res $\mathrm{J}$. 1995;21(4):487-504.

DOI: $10.1080 / 0141192950210404$.

19. Gregg P, Machin S. The relationship between childhood experiences, subsequent educational attainment and adult labour market performance. In: Vleminckx K, Smeeding T, editors. Child well-being in modern nations: what do we know? Bristol: The Policy Press; 2001.

20. Dewald JF, Meijer AM, Oort FJ, Kerkhof GA, Bögels SM. The influence of sleep quality, sleep duration and sleepiness on school performance in children and adolescents: a meta-analytic review. Sleep Med Rev. 2010;14(3):179-89.

DOI: 10.1016/j.smrv.2009.10.004

21. Singh A, Uijtdewilligen L, Twisk JWR, van Mechelen W, Chinapaw MJM. Physical activity and performance at school: A systematic review of the literature including a methodological quality assessment. JAMA Pediatr. 2012;166(1):49-55.

DOI: $10.1001 /$ archpediatrics.2011.716

(c) 2016 Richards and Smith; This is an Open Access article distributed under the terms of the Creative Commons Attribution License (http://creativecommons.org/licenses/by/4.0), which permits unrestricted use, distribution, and reproduction in any medium, provided the original work is properly cited.

Peer-review history:

The peer review history for this paper can be accessed here: http://sciencedomain.org/review-history/15325 